\title{
Research on Software Development Method Based on SOA Architecture
}

\author{
Saie Liu*, Chang Xia \\ Yunnan Vocational College of Land and Resources, 650500, China
}

Keywords: Web Services, SOA System, Teaching Management, Secondary Development.

\begin{abstract}
With the development of science and technology and the emergence of Web services, a new SOA architecture has been widely used, which can better complete software development. On this basis, this paper analyzes the software development method of SOA architecture. Firstly, the characteristics and development advantages of SOA architecture are briefly summarized; secondly, through specific application examples, specific SOA Software development methods are summarized; finally, the development of teaching management system based on SOA is introduced.
\end{abstract}

\section{Introduction}

The service system based on SOA Software development needs to be constructed from three aspects: service consumer, service provider and service publisher. Service systems play different roles in the structure. Service center plays the role of request execution and function optimization. Although the goal is set before software development, the specific structure optimization may be affected by the network operating environment. In order to determine the goal of the service provider, the programming language written in the software development process can play a link role in the structure, and the actual development goal also represents the role of the circuit in the actual software operation. If the software development process needs to continue to optimize the functional design, it needs to continue to optimize the set development goals. In a service-oriented architecture component, developers' functional goals and expected functions should also be set. Software goals can be combined with each other when they are implemented, but for architecture development problems. Strict distinction will be made on the functional objectives, and the corresponding database will remain stable in the process of functional realization[1]. In the structural functional verification guided by service architecture, the functional objectives will focus on the software development stage and the mutual control and connection between service architectures.

\section{Software Development Method Of Soa Architecture}

Architecture based software program components can be built with a single configuration. Furthermore, a plurality of assembly language programs can be coupled to form software that is executing programs. The software program server of the accessed object describes the target and integrates the described data information according to the function[2]. As a result, software program editing can effectively update and obtain data information of network environment. The service architecture is based on the business requirements and software program construction technology that can realize more efficient service system deployment. Through the mutual control between the program assembly and various functions, the mutual combination between the application of the software assembly language and the functional structure system will inevitably take place between the structure and the functional decision. In order to realize the function connection of software development through program assembly, the optimization mode needs to be determined. The program assembly system based on the structure system can design the overall structure of the field, which can not only save time for program configuration, but also avoid the mutual interference of programs. 


\section{SOA Architecture}

\subsection{SOA Software Development Method}

In the construction of the construction system, the method of developing and using the software is decided first. Software development is also based on objective functions. Select the basic service equipment to build the structure system. This can sort out the user's various use needs, clarify the access direction, and establish the software program database on the network. Based on the database wizard, users can meet their own needs when querying software information[3]. Consumers play the role of setting goals in software development, and the actual functions are also reflected in the structural system. However, there are some hidden necessary conditions in the actual development process. Due to this requirement, the software is not reflected in the normal state. In order to make the software reach the best state in operation, it is necessary to perform the database press control of the hidden function. That is, in the network connection, the hidden database setting needs to be set to the software development state. Once the information obtained in the network environment matches the hidden function, in order to understand the corresponding function and control selection, it is easier for users to think of the software. Software development method is the key to realize the following functions. In any state, SOA architecture is very different from traditional software. It is more powerful and can meet the needs of different directions[4]. In this software development, because the purpose of use is initially determined by the assembly of the software program, it can adapt to different application directions in the development stage. Software development and structural system decisions need to reflect multiple directions.

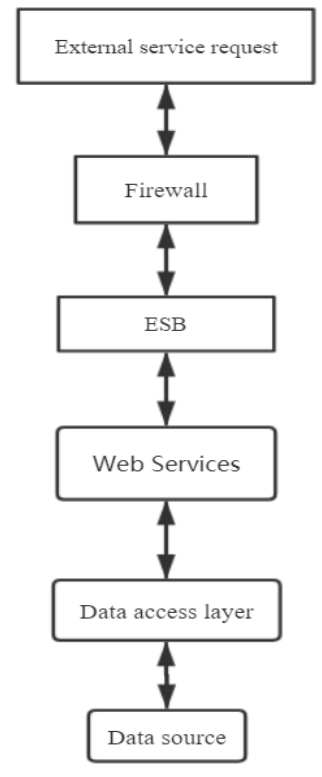

Figure 1 Architecture of computer teaching management system

\subsection{SOA Function Implementation Technology}

The implementation of SOA functions is based on existing key technologies. The existing technology mainly integrates software functions into the system. The identified functions are integrated and integrated into the integrated database through integration. In the existing functional technology and software control process, there are database docking obstacles, there are docking obstacles. This method can be further improved. The implementation of this function needs to establish a standardized framework. Especially in the stage of network interface selection, it is necessary to design and develop in the network environment in accordance with the use of security standards[5]. Development and design is to ensure the download speed of data information, not only between the development stages, to avoid the potential risk of quality and safety, the normal operation of the server has an impact, so information download in the network environment must be avoided. Based on the construction of SOA, the developed software can be used in the state of 
network access. In addition, you can start the normal function in the broken link state. The classification of database and the division of building modules are the main technologies of developing software, such as image publishing and service description of discovery services, etc. the network transmission information uses HTTP, SMTP and FTP technologies. WSDL is also a technical method related to development. This technology can choose development language, be in the state of network server connection, and combine the information in the program in the network information acquisition environment.

Software programs built on SOA architecture need an adaptive environment to implement functions. The key technology to realize SOA is to build the working environment[6]. All functions can be realized in the network environment. Some information of the software program needs to be accessed through the network platform. But it also requires existing functionality. In the broken state, activate some existing functions and save the data generated in the software operation locally. UDDI technology is used to define the form of following SOA software program operations. Users can customize various forms when operating software functions, such as defining the operation mode and information acquisition mode of web server. Software has different functions, and the form of server functions often changes, but the final optimization technology has obvious integration. In the software application technology management, after selecting the operation method, it can effectively query the login information[7]. Due to various problems in the process of technical application, the server public file can also effectively describe it. The general problem is that when the network is connected, you can repair it by yourself, but some more complex problems, programs, are being executed. When they are being executed, they remind the operators of the problem of manual operation. After understanding the problem, it is necessary to deal with it. The selected operation method will be updated after problem research and synthesis, and the operation method can be selected by combining multiple technologies. However, the security of data in use must always be given priority. Only to ensure the security of the database, without affecting the realization of various functions.

\section{SOA Application Instance}

\subsection{Application of Computer Teaching Management System}

SOA architecture and component software programs are most commonly used to teach management systems. SOA Software Technology is used in the teaching management system, which is established for the realization of distance teaching without time and place constraints[8]. It is very convenient to analyze student performance data. In distance education, the perfect structure system is reflected in the overall level of technology application. SOA architecture is characterized by wide area distribution. CMI system can be used to analyze all kinds of data produced in the teaching process and extra components of the teaching process, including the parts that need to be further improved in distance education. Through the construction of distance education system, the management system can meet the application requirements in different situations, and can carry out the routine division of teachers' teaching function and students' learning function. The computer teaching management system can be used in normal teaching state. In the development of software learning, it can be divided into the elements of different problems with students' learning. Computer education management has independent learning function. According to the different functions proposed in the operation in the learning state, the current software operation status can be changed. In the case of more efficient control management, SOA structure is used to teach management software, and the software functions are more diverse[9]. Different from the existing technology, the most remarkable feature of this technology is the real-time popularization of information and data. In the process of developing distance education curriculum, the segmentation of the structured curriculum can avoid the delay or pause of audio education information. In the national standards of distance education, the following requirements are designated as the interoperability of Education Management: the same course can be used in different CMI systems; the programs that need to be developed on different computers must be combined in CMI systems; the development route of one 
CMI system can be moved to another CMI system. Students' data can be analyzed simply.

\subsection{Design of CMI System Based on SOA}

In addition to computer education management system, SMI system can also be designed based on SOA technology. First, the external service request is constructed, and the firewall department is set as security. Information requests that ensure the absolute security of data sources can enter the ESB stage after testing, that is, the internal request stage of the server[10]. The start-up function of the data access layer is completed through the real-time connection between the internal request and the database. After data access, the final data source is exit. User interface, interaction and function among them, and monitoring and control function of firewall on main functions. WsServices constructs the user program composition structure, and enters the final integration stage in the structure system according to various requirements. After the configuration of the system is constructed and the direction of the software application is determined, the function can be used to enter a more secure state, especially in the software function integration stage. In the network environment, it can meet different needs and enter a more secure state.

\section{Conclusion}

SOA represents the emergence of standardized, loosely coupled and rough software architecture, as well as new software development ideas that are not constrained by specific technology implementation. Of course, the technology of SOA services has reached SOA. It plays an important role in the process of maturity. "From the perspective of SOA, emphasizing service in different platforms and development environments is one of the several choices of current distributed technology. This article is about the current state of SOA and the background of its advantageous architecture. Introduced and analyzed the implementation methods and other aspects! Also introduced as SOA installation technology services. Finally, the service is illustrated in detail with specific examples of SOA development. Thanks to the unique advantages of SOA, it conforms to the trend of software development in the future, and will become the new generation mainstream of thorough research and fierce competition among major software suppliers.

\section{Acknowledgements}

Design and implementation of online examination SYSTEM-A case study of Yunnan Vocational College of Land and Resources. Serial number: GTZJ-2017-34, Source: Land and Resources Staff Education Research Association, Vocational Education

\section{References}

[1] Mitric, C., Leung, S. O. A., Monton, L., et al. O-OBS/GYN-EDU-209 - Substituting Lectures with Interactive Teaching in OBGYN Clerkship: Integrating Hands-on Learning with Theory, vol. 40, no. 6, pp. 845-846, 2018.

[2] Bregonziorozier L, Giorio C, Siekmann F, et al. Secondary organic aerosol formation from isoprene photooxidation during cloud condensation-evaporation cycles, Vol. 16, pp. 1747-1760, 2017.

[3] Jianlin, Hu., Shantanu, Jathar., Hongliang, Zhang., Long-term particulate matter modeling for health effect studies in California - Part 2: Concentrations and sources of ultrafine organic aerosols. Atmospheric Chemistry \& Physics, vol. 17, no. 8, pp. 5379-5391, 2017.

[4] Zhang, Y., Tang, L., Sun, Y., et al. Limited formation of isoprene epoxydiols - derived secondary organic aerosol under NOx - rich environments in Eastern China. Vol. 44, no. 4, pp. 2035-2043, 2017.

[5] Deming, Han., Zhen, Wang., Jinping, Cheng., Volatile organic compounds (VOCs) during non- 
haze and haze days in Shanghai: characterization and secondary organic aerosol (SOA) formation. Environmental Science \& Pollution Research, vol. 24, no. 4, pp. 1-11, 2017.

[6] Pieber, S.M, Kumar N K, Klein F, et al. Gas-phase composition and secondary organic aerosol formation from standard and particle filter-retrofitted gasoline direct injection vehicles investigated in a batch and flow reactor, pp. 1-41, 2018.

[7] Qi, Chen., Tzung-May, Fu., Jianlin, Hu., Modelling secondary organic aerosols in China. National Science Review, vol. 4, no. 6, pp. 806-809, 2017.

[8] Songkiat, Chantarogh., Soamarat, Vilaiyuk., Thipwimol Tim-Aroon,Clinical improvement of renal amyloidosis in a patient with systemic-onset juvenile idiopathic arthritis who received tocilizumab treatment: a case report and literature review. Bmc Nephrology, vol. 18, no. 1, pp. 159, 2017.

[9] Meng, J.J., Hou, Z.F., Liu, X.D., et al. Compositions and sources of summertime dicarboxylic acids and related SOA in PM2.5 from Mt. Taishan, 2017.

[10] Nima, Afshar-Mohajer., Barron, Henderson. How does a 10-fold pulse increase of aircraftrelated NOx impact the global burdens of $\mathrm{O} 3$ and secondary organic aerosol (SOA). Air Quality Atmosphere \& Health, pp. 1-10, 2017. 